\title{
Observation of "Diffusion Modes" in Measurement of the Diffusion Coefficient of Cesium in Noble Gases
}

\author{
P. RUDECKI \\ Institute of Physics, N. Copernicus University \\ Grudziądzka 5-7, 87-100 Toruń, Poland
}

(Received August 3, 2001)

\begin{abstract}
A set of experiments investigating diffusion of alkali atoms in the noble gases is reported. A cesium gradient in the cell was induced by electrodeless pulse discharge. In the transient signal of absorption, corresponding to the ${ }^{2} S_{1 / 2}-n^{2} P_{J}$ transitions, five diffusion modes were observed. The diffusion coefficient $D$ of cesium in helium and argon has been measured in a spherical cell filled with a buffer gas of 2.5 to $30 \mathrm{hPa}$ and for temperatures in the range from $275 \mathrm{~K}$ to $400 \mathrm{~K}$.
\end{abstract}

PACS numbers: $82.56 . \mathrm{Lz}, 34.90 .+\mathrm{q}$

\section{Introduction}

Modelling the low-pressure alkali-noble gas atom discharge in various plasma devices needs to include the parameters crucial for description of the transport processes such as ambipolar diffusion as well as ground and excited state diffusion.

Several experiments can provide information about the diffusion coefficient. An optical observation of the evolution of the spatial distribution of atoms created in a pulse electrical discharge has been studied with an increasing interest during the last few decades. Since the studies done by Phelps and Molnar [1] experimental investigations have dealt mainly with quenching and transport processes of metastable species. The principle of the experimental method for atoms in their ground state was described by Cornellissen [2], who developed a pulse glow discharge to create the gradient of ground state atoms in a long cylindrical cell. 
An optical observation of optically polarized ground state atom is also a tool for investigating the diffusion [3-5]. Unfortunately, in optical pumping experiments the diffusion can be observed in the presence of dominant spin relaxation processes.

New laser techniques, such as light-induced diffusive pulling (LDP) [6] and light induced drift (LID) [7] are restricted due to experimental condition. The LDP and LID experiments are carried out for the pressure greater than $100 \mathrm{hPa}$ and less than $15 \mathrm{hPa}$, respectively. An investigation of temperature dependences is also limited.

The optical methods base on description of an evolution of the system by the diffusion equation with a linear relaxation term. The solution of the diffusion equation is given as an infinite sum of exponential terms (commonly called diffusion modes) and it is difficult to fit the signal to the theoretical description. An approximation of the signal consisting in only one diffusion mode appears to be unrealistic in many experimental situations, especially in the measurement of the diffusion coefficient.

In the present set of experiments, the diffusion coefficient is measured using electrodeless pulse discharge to create a gradient of cesium atoms. A chosen geometry of discharge allows us to limit the number of diffusion modes in the observed signal of absorption. On the other hand, a possibility of observation of a few or even one mode signal in optical pumping experiments was analysed for a spherical cell by Legowski and the author [8] and independently by Bernabeu and Tornos [9] for pure spherical symmetric distribution of atoms in the cell. This procedure is used in the present work to analyse the absorption signals.

\section{Experimental details}

The experiments were performed for a spherical cell, which is placed in the high frequency discharge coils. Two geometries of the coils, which were used in the experiments, are shown in Fig. 1, where they are denoted by " $A$ " and " $B$ ". The pulse of electrodeless r.f. discharge (about $90 \mathrm{MHz}$ ) produces a strong cesium gradient. After switching off the pulse discharge, the spatial density of cesium atoms evolves to a homogeneous distribution.

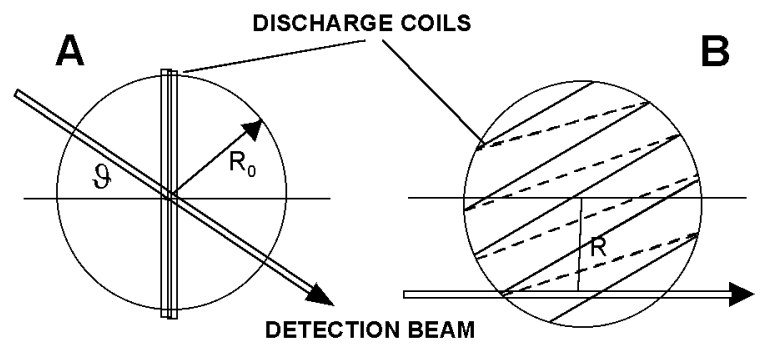

Fig. 1. Geometry of the discharge and the detection. 
The symmetry of the spatial distribution of the cesium atoms $N(r, t)$ is assumed to be axially symmetric since it is guaranteed by the geometry of the discharge coils. The evolution of atoms for axially symmetric initial spatial distribution is characterised by the inversion symmetry with respect to the centre of spherical cell for the "A" type discharge. For the "B" type of discharge spherical symmetric distribution of atoms is expected. The transient signal of absorption due to $6^{2} S_{1 / 2}-n^{2} P_{J}$ transitions (with $n=6,7$ or 8 to reduce the optical thickness) was observed after the pulse discharge. Then, the absorption signal of the detection beam is compared to the solution of the diffusion equation corresponding to the experimental condition. For the above assumptions the evolution of the cesium ground state density $N(r, \vartheta, \varphi, t)$ can be described in spherical coordinate system with the boundary condition $N\left(r=R_{0}\right)=N_{W}$ (the density at the wall is assumed to be constant) and $\partial N / \partial \varphi=0$ by the following sum of the diffusion modes:

$N(r, \vartheta, t)=N_{W}-\sum_{k, m=0}^{\infty} A_{2 k, m} J_{2 k+1 / 2}\left(\Omega_{2 k, m} r\right) \frac{1}{\sqrt{r}} P_{2 k} \cos \vartheta \exp \left(-D \Omega_{2 k, m}^{2} t\right)$,

where $D$ is the diffusion constant and $\Omega_{i, m}$ is a geometrical factor which depends on the geometry of the cell and a boundary condition on the cell walls. Here $J_{i+1 / 2}\left(\Omega_{i, m} r\right)$ are the Bessel functions and $\Omega_{i, m}$ are given as positive roots of the following equation:

$$
J_{i+1 / 2}\left(\Omega_{i, m} R_{0}\right)=0 .
$$

Here $P_{i}(\cos \vartheta)$ are the Legendre polynomials; $R_{0}$ is the inner radius of the cell.

The coefficient $A_{i, m}$ depends on the following initial condition:

$$
\begin{gathered}
A_{2 k, m}=\frac{4 k+1}{R_{0}^{2}\left[J_{2 k+1 / 2}^{\prime}\left(\Omega_{2 k, m} R_{0}\right)\right]^{2}} \\
\times \int_{0}^{\pi} \int_{0}^{R_{0}}\left[N_{W}-N(r, \vartheta, t=0)\right] r^{3 / 2} J_{2 k+1 / 2}\left(\Omega_{2 k, m} r\right) P_{2 k} \cos \vartheta \sin \vartheta \mathrm{d} \vartheta \mathrm{d} r .
\end{gathered}
$$

The experimental arrangement is shown in Fig. 2.

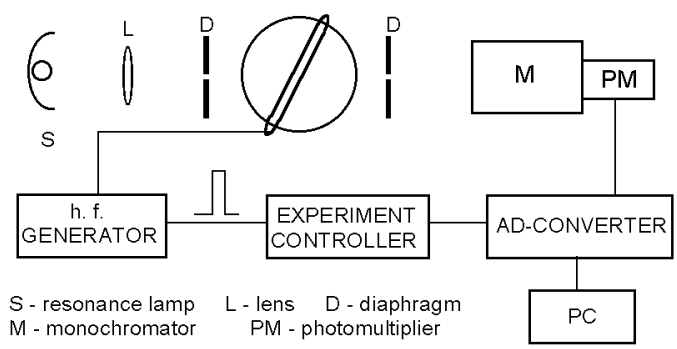

Fig. 2. Experimental set-up. The discharge cell is located in an oven. For the "A" type experiment the cell was rotated and for the " $\mathrm{B}$ " type shifted. 
The source $S$ is a high frequency discharge resonance lamp for an optical excitation. The signal was converted by an AD-converter. The digital signal was averaged and stored in the computer. The number of runs to be averaged was from 20 to 200. The diameter of the detection beam was $0.6 \mathrm{~cm}$, and the pulse time of the discharge was from 0.1 to $10 \mathrm{~ms}$. The intensity and repetition period of the r.f. pulse discharge were varied. The cells were carefully selected pyrex bulbs. The inner radius $R_{0}$ of the cells was about $4 \mathrm{~cm}$. The cells were heated to eliminate the impurities that could have been present on/in pyrex walls. Metallic cesium was distilled under high vacuum into the cell at room temperature and then an ultrapure buffer gas was admitted. The cell was disconnected from the vacuum system. The above procedure was used to ensure an appropriate condition of electrodeless discharge at all buffer gas pressures and all temperatures of the experiment. The cell was heated or cooled by temperature-regulated air flow.

The total signal for a small optical thickness can be represented by

$$
S(t)=S(\infty)-\sum S_{i, m} \exp \left(-Z_{i, m} t\right) \propto \int_{V} N(\boldsymbol{r}, t) \mathrm{d} \tau
$$

where $Z_{i, m}=D \Omega_{i, m}^{2}$ denotes a diffusion mode decay constant, $V$ is a volume of the cell touched by the detection beam. It should be noted that for thin detection beam and an experiment of the "A" type we have [8]

$$
S_{i, m} \propto A_{i, m} P_{i}(\cos \vartheta) \alpha_{i, m},
$$

where

$$
P_{0}=1, P_{2}=\left(3 \cos ^{2} \vartheta-1\right) / 2, \ldots,
$$

and

$$
\alpha_{i, m}=\int_{V} J_{i+1 / 2}\left(\Omega_{i, m} r\right) r^{-1 / 2} \mathrm{~d} \tau
$$

For the "B" type experiments, i.e. in the case of the spherical symmetrical distribution of atoms, the $S_{0, m}$ terms appear only in the signal.

\section{Results and discussion}

The transient signals for the " $\mathrm{A}$ " and the " $\mathrm{B}$ " types of the experiments performed in the course of the present study are shown in Figs. 3 and 4.

Only the first few $S_{i, m}$ terms contribute to the signal and only five of these (with $i=0, m=0,1,2$ and $i=2, m=0,1$ ) are considered. The quantities $\Omega_{i, m}$ have increasing relative values (see Fig. 5) and a higher modes decay too fast to be observed. Only three exponential curves could be well fitted to the transient signal with a good accuracy and thus the non-radial terms $(i=2, m=0,1)$ were extracted from normalised to the $S_{0,0}$ difference $S\left(\vartheta=90^{\circ}\right)-S(\vartheta=0)$ of the signals. This way of the signal analysis leads to smaller errors for $D$ and $S_{i, m}$ because the values of $Z_{0,1}$ and $Z_{0,2}$ are close to the $Z_{2,0}$ and $Z_{2,1}$ respectively 

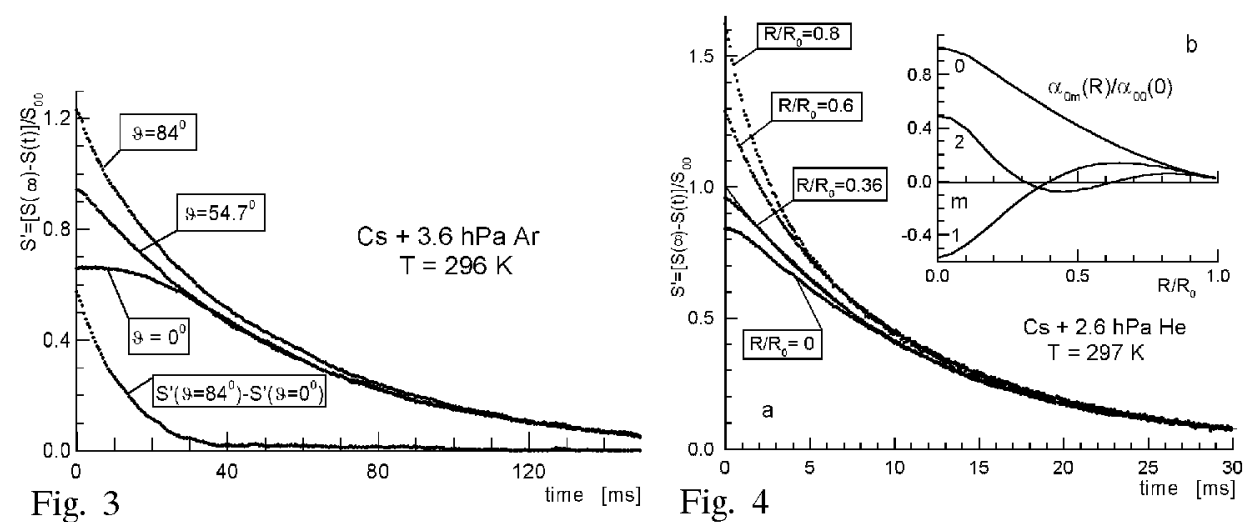

Fig. 3. The signals for the "A" type of the experiment.

Fig. 4 The signals for the "B" type of the experiment. (a) The solid line fits one exponential curve. The normalised $\alpha_{0, m}$ integrals are given in (b).
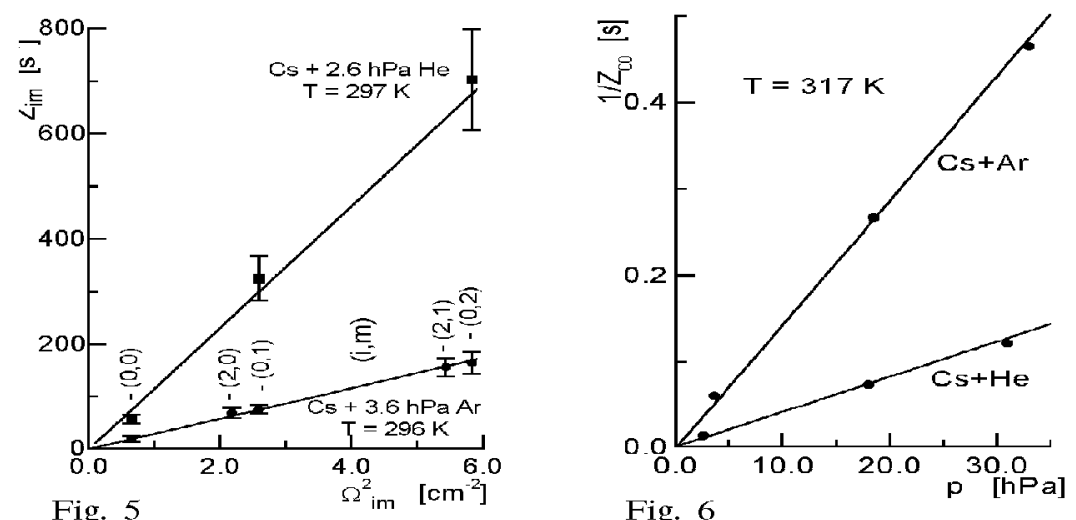

Fig. 6

Fig. 5. $Z_{i m}$ against discrete values of $\Omega_{i m}$ which fulfil the assumed boundary condition. $Z_{i m}$ have been fitted here as independent parameters.

Fig. $61 / Z_{00}$ against the pressure of the buffer gases. The results have been obtained from the "B" type experiments at $R / R_{0}=0.36$.

and then the pairs of the fitting parameters $S_{0,1}, S_{2,0}$ and $S_{0,2}, S_{2,1}$ are strongly correlated. The analysis of the $Z_{i m}$ values and qualitative relations between $S_{i, m}$ amplitudes are consistent with the above theoretical description. One can show that the sign of the $S_{2, m}$ amplitudes changes as $P_{2}(\vartheta)$ in the "A" type experiment as well the $S_{0, m}$ as $\alpha_{0, m}$ (see Fig. $4 \mathrm{~b}$ ) for the "B" one. In good agreement with theoretical prediction [8] the signal fits by just one exponential curve for $R / R_{0}=0.36$. A least-squares fitting approximates then the $Z_{0,0}$ values with an accuracy better than $2 \%$. Quasi-isotropic discharge gives practically a spherical 
symmetrical distribution of cesium atoms. In Fig. $5 Z_{i, m}$ are shown. In the figure the $\Omega_{i m}$ values fulfil exactly Eq. (2). The slope of the fitted straight line gives the $D$ value. For an exact estimation of the diffusion constant the $D$ and $S_{i, m}$ quantities were used as the fitting parameters.

One can use this method to measure the diffusion coefficient in a wide range of the buffer gas pressure as is illustrated in Fig. 6. The results of the systematic measurements of the temperature dependence of the diffusion coefficient have been carried out at spherical symmetry of the density gradient of the cesium atoms. The pressure of the buffer gases (at filling temperature of about $296 \mathrm{~K}$ ) was 2.6, 18.0, $31.2 \mathrm{hPa}$ for $\mathrm{Cs}_{\mathrm{s}}-\mathrm{He}$ system and $3.6,18.5,33.1 \mathrm{hPa}$ for $\mathrm{Cs}-\mathrm{Ar}$ system.

The experimental values of the diffusion coefficient were compared with those calculated using theoretical potentials. The theoretical calculations are based on the classical Hirschfelder and Chapman-Enskog approximation $[10,11]$ (see Appendix). Such calculations are reliable at a high temperature range. Here we have used for calculations the numerical potentials of Czuchaj and Sienkiewicz [12], Pascale and Vandeplanque [13] and Buck and Pauly [14]. The final experimental and theoretical results are given in Fig. 7 .

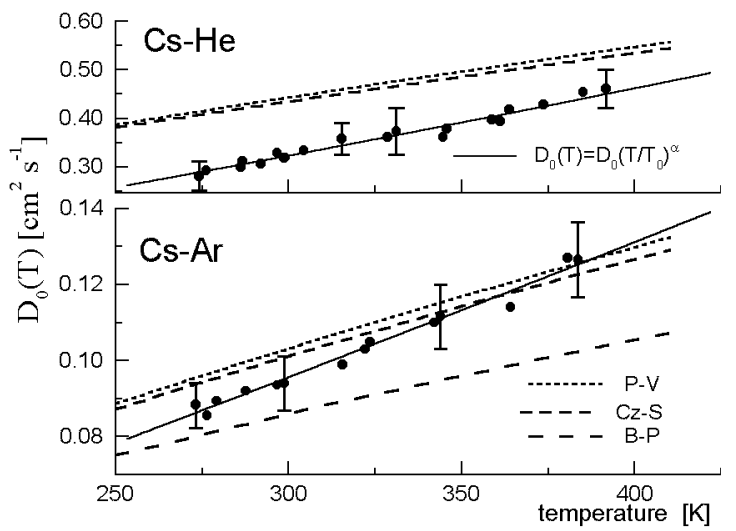

Fig. 7. The final experimental and theoretical results. The calculations at Czuchaj and Sienkiewicz, Pascale and Vandeplanque and Buck and Pauly are denoted as $\mathrm{Cz}-\mathrm{S}, \mathrm{P}-\mathrm{V}$ and B-P.

A brief comparison of experimental values for $D_{0}$, i.e. the value of $D$ at the normal pressure, with the values obtained in optical pumping experiments as well as with the theoretical ones is shown in the Table assuming a power-law temperature dependence for $D_{0}(T)$

$$
D_{0}(T)=D_{0}\left(T / T_{0}\right)^{\alpha} .
$$

It should be noted that Eq. (6) describes analytically the character of temperature dependence of diffusion coefficient for pure repulsive potential of the 
TABLE

Experimental and theoretical diffusion coefficient. Other experiments have been extrapolated as $D_{0}(T)=D_{o}\left(T / T_{0}\right)^{\alpha}$ at $\alpha=1.5 . D_{0}$ is in $\mathrm{cm}^{2} \mathrm{~s}^{-1}$.

\begin{tabular}{|c|c|c|c|c|c|}
\hline \multirow[t]{2}{*}{ Gas } & \multicolumn{2}{|c|}{ Present measurement } & Other results & \multicolumn{2}{|c|}{ Theory } \\
\hline & $D_{0}$ & $\alpha$ & $D_{0}$ & $D_{0}$ & $\alpha$ \\
\hline \multirow[t]{2}{*}{$\mathrm{He}$} & $0.295(21)$ & $1.28(26)$ & $0.34(3)^{\mathrm{B}}$ & 0.408 & $0.72^{\mathrm{Cz}-\mathrm{S}}$ \\
\hline & & & $0.291(30)^{\mathrm{F}-\mathrm{S}}$ & 0.414 & $0.73^{\mathrm{P}-\mathrm{V}}$ \\
\hline \multirow[t]{3}{*}{ Ar } & $0.086(5)$ & $1.12(16)$ & $0.15(5)^{\mathrm{B}}$ & 0.094 & $0.79^{\mathrm{Cz}-\mathrm{S}}$ \\
\hline & & & $0.111(11)^{\mathrm{F}-\mathrm{S}}$ & 0.096 & $0.82^{\mathrm{P}-\mathrm{V}}$ \\
\hline & & & $0.101(10)^{\mathrm{T}-\mathrm{A}}$ & 0.080 & $0.73^{\mathrm{B}-\mathrm{P}}$ \\
\hline
\end{tabular}

inverse-power $C / R^{n}$ type. One can see that these calculations provide an interesting comparison with experimental measurement. The agreement is quite good for the Cs-Ar system and rather poor for the $\mathrm{Cs}-\mathrm{He}$ one. Only the absolute value of the $D_{0}$ was charged by a systematic correction (for example, measurements of the buffer gas pressure or deviation from pure spherical symmetry of the bulb). In the present experiment this correction is considered to be less than $5 \%$. Hence, we limit ourselves to the comparison between experimental and theoretical values of $\alpha$ coefficient, which is not disturbed by the systematical uncertainties. The significant differences are observed here.

The exact formula for Lennard-Jones' $(n-m)$ type of a potential [11] is as follows:

$$
D_{0}(T)=D^{*}\left(\frac{T}{T_{0}}\right)^{0.5+2 / n} \frac{T^{(n-m) / n}}{\beta+T^{(n-m) / n}}
$$

with constants $D^{*}$ and $\beta$. This formula contains too many free parameters to be representative for description of the experimental results. It means that the experimental results are sensitive to rather a small region of a repulsive branch of the potential and a wide class of the Lennard-Jones type of potentials could be fitted to experimental data. A reliable approximation of the measurements by Eq. (6) implies only that the theoretical potentials are too much repulsive.

\section{Conclusion}

A new set of experiments for accurate determination of the diffusion coefficient was performed. While the experimental studies have been restricted to the alkali-metal in noble gas, the technique can be applicable to other atoms in 
the ground state. Similar experiments can be applied to investigate diffusion of excited-metastable atoms. These promising experimental ideas can be also very useful in examining the spatial distribution of atoms in a spherical cell.

In view of the light-induced diffusive pulling and light-induced drift experiments, which concern diffusion measurements $[6,7]$, the method described in the present work seems to be particularly suited to measure independently the diffusion coefficient of the alkali atoms in the ground state.

The calculation of the diffusion coefficient was carried for the available potentials. Although some of the available potentials may not be very accurate, the calculations allow us to have a wide view of reproduced experimentally and theoretically diffusion data at various temperatures. The author is convinced that the diffusion coefficients presented here are more reliable than the other experimental data, however a detailed comparison of the experimental and theoretical results requires measurements performed in a wide range of temperatures. Theoretical calculations should be then performed using quantum mechanical methods and more realistic potentials.

\section{Acknowledgment}

The author wishes to thank Prof. J. Szudy for critical reading the manuscript of this paper.

\section{Appendix}

In the Chapman-Enskog [10] approximation for binary mixture the diffusion constant is given by

$$
D=\frac{3}{16} \sqrt{\frac{2 \pi k T}{\mu}} \frac{k T}{p} \frac{1}{Q_{d}}
$$

with $p$ - total peturber pressure, $\mu$ - the reduced mass and $Q_{d}$ - energy averaged cross-section as

$$
Q_{d}=\int_{0}^{\infty} g^{5} \exp \left(-g^{2}\right) Q^{\prime}(v) \mathrm{d} g
$$

where

$$
g^{2}=\mu v^{2} / 2 k T
$$

and

$$
Q^{\prime}(v)=2 \pi \int_{0}^{\infty}[1-\cos \chi(v, b)] b \mathrm{~d} b
$$

Here $v$ is the initial relative speed of colliding atoms, $b$ is the impact parameter and $\chi$ is the deflection function

$$
\chi(v, b)=\pi-2 b \int_{r_{m}}^{\infty} \frac{\mathrm{d} r}{r^{2}\left[1-b^{2} / r^{2}-2 \varphi(r) / \mu v^{2}\right]^{1 / 2}} .
$$

In Eq. (A4) $r_{m}$ denotes the distance of the closest approach and $\varphi(r)$ is the intermolecular potential energy. 


\section{References}

[1] A.V. Phelps, J.P. Molnar, Phys. Rev. 87, 1202 (1952).

[2] H.J. Cornelissen, J. Phys. B, At. Mol. Opt. Phys. 18, 3445 (1985).

[3] F. Bylicki, Bull. Acad. Pol. Sci. Ser. Sci. Math. Astronom. Phys, 26, 945 (1978).

[4] F.A. Franz, C.E. Sooriamoorthi, Phys. Rev. A 10, 126 (1974).

[5] J. Tornos, J.C. Amare, Appl. Spectrosc. 40, 596 (1986).

[6] A.P. Hickman, D.T. Mugglin, A.D. Streater, Opt. Commun. 102, 281 (1993).

[7] F. Yahyaei-Moayyed, A.P. Hickman, A.D. Streater, J. Phys. B, At. Mol. Opt. Phys. 29, 435 (1996).

[8] S. Legowski, P. Rudecki, Bull. Acad. Pol. Sci. Ser. Sci. Math. Astronom. Phys. 18, 43 (1970).

[9] E. Bernabeu, J. Tornos, J. Opt. Soc. Am. B 1, 586 (1984).

[10] J. Hirschfelder, C. Curtis, R. Bird, Molecular Theory of Gases and Liquids, Wiley, New York 1964

[11] S. Chapman, T.G. Cowling, The Matematical Theory of Non-Uniform Gases, Cambridge Press, Cambridge 1970.

[12] E. Czuchaj, J. Sienkiewicz, Z. Naturforsch. A 34, 694 (1979).

[13] J. Pascale, J. Veplanque, J. Chem. Phys. 60, 2278 (1974).

[14] U. Buck, H. Pauly, Z. Phys. 208, 390 (1968). 